\title{
End-functionalized block copolymer electrolytes: effect of segregation strength on ion transport efficiency
}

\author{
Gyuha Jo ${ }^{1}$, Onnuri Kim ${ }^{1}$, Hoon $\mathrm{Kim}^{1}$, U Hyeok Choi ${ }^{2}$, Sang-Bok Lee ${ }^{2}$ and Moon Jeong Park ${ }^{1,3}$
}

We report the synthesis, morphology and ion transport properties of poly(styrene-b-ethylene oxide) (PS-b-PEO) block copolymers with various end-functional groups. The PS-b-PEO having an - $\mathrm{OH}$ terminal group showed poorly defined morphology, while the inclusion of a terminal acid unit $\left(-\mathrm{SO}_{3} \mathrm{H}\right.$ or $\left.-\mathrm{COOH}\right)$ in PS-b-PEO caused the development of an ordered lamellar structure. The terminal acid moieties in PEO were found to increase the segregation strength of PS-b-PEO and reduce the degree of crystallinity of PEO. This observation is intriguing given that the end group concentration of PEO in PS-b-PEO employed in this study is as small as 0.4 mol\%. Decreased conductivity was observed for PS-b-PEO tethered with acid groups when doped with lithium salts, which we ascribe to the slow segmental motion of acid group-terminated PEO chains. However, organization of the PEO domains of PS-b-PEO with terminal acid units into self-assembled nanostructures having sharp interfaces proved beneficial for increasing ion transport efficiency by creating less tortuous ion conduction pathways. At high levels of salt loading, the thermodynamic effects of the terminal group on ion transport properties of PS-b-PEO were attenuated. Our results suggest a unique methodology for controlling the morphology and ion transport properties of block copolymer electrolytes via attachment of a single end functional unit.

Polymer Journal (2016) 48, 465-472; doi:10.1038/pj.2015.141; published online 20 January 2016

\section{INTRODUCTION}

In recent years, there has been increasing demand for highly efficient polymer electrolytes in the solid state for use in advanced lithium batteries. ${ }^{1,2}$ This demand is due to the inherent drawbacks of liquid electrolytes at high cell potentials, such as flammability and decomposition reactions. ${ }^{3,4}$ To this end, poly(ethylene oxide) (PEO)-based polymers have been extensively investigated over the past decades because of the high ionic conductivity of $\mathrm{PEO} /$ lithium salt complexes. $^{5-8}$ However, the poor mechanical stability of PEO does not prevent lithium dendrite formation at the interfaces between anode and electrolyte, which causes unexpected short-circuits of the cells. ${ }^{7,8}$

One appealing solution to improve the mechanical properties of PEO polymers is the incorporation of mechanically robust polymer chains, such as polystyrene (PS), ${ }^{9-18}$ polyethylene, ${ }^{19,20}$ polysiloxane, ${ }^{21,22}$ polymethacrylate ${ }^{23}$ and polythioamide. ${ }^{24}$ However, such hard polymers are essentially insulators to lithium ions, and the inevitable result of increasing mechanical strength in this manner is reduction in ionic conductivity. Hence, a wide variety of strategies for combining PEO with hard polymers have been proposed to achieve the synergistic goals of improving conductivity while maintaining mechanical integrity. ${ }^{9-13}$ Examples include the creation of microphase-separated morphologies by designing the polymers with block or graft architecture, as pioneered by Balsara and coworkers. ${ }^{10,11}$
While such research efforts have opened new prospects in the era of high-conductivity solid-state electrolytes, defined as having a conductivity of $10^{-6}$ to $10^{-4} \mathrm{~S} \mathrm{~cm}^{-1}$ at room temperature, ${ }^{9-11,15,24}$ fundamental flaws of high charge-transfer resistance at the electrode/ electrolyte interfaces are encountered for the development of high performance batteries. ${ }^{25-27}$ In addition, coexisting non-conducting hard polymers influence lithium-ion diffusion across PEO domains due to interfacial mixing, ${ }^{14,15}$ which leads to lower lithium transport rates than predicted. These problems have emerged as important challenges for advancing battery technologies, but the knowledge to overcome these hurdles is lacking.

These problems may be resolved by preparing PEO polymers end-functionalized with anionic moieties to expedite lithium transfer at electrode/electrolyte interfaces, and to form passivation layers. ${ }^{28,29}$ The modification of PEO chains with ionic units is also expected to decrease thermodynamic compatibility between PEO and ionophobic hard polymers. This will help create sharp interfaces in the microphase-separated morphologies. ${ }^{24,30}$

To these ends, we recently reported the synthesis of a set of poly (styrene-b-ethylene oxide) (PS-b-PEO) block copolymers, in which the terminal group of $\mathrm{PEO}$ chains was synthesized as either $-\mathrm{OH}$, $-\mathrm{SO}_{3} \mathrm{H}$ or $-\mathrm{SO}_{3} \mathrm{Li}^{30}$ The alteration of a single end group to sulfonic acid in PEO of PS-b-PEO resulted in remarkable changes in the type of self-assembled morphology, ascribed to intermolecular interactions

\footnotetext{
${ }^{1}$ Department of Chemistry, Pohang University of Science and Technology (POSTECH), Pohang, Korea; ${ }^{2}$ Functional Composites Department, Korea Institute of Materials Science (KIMS), Changwon, Gyeongnam, Korea and ${ }^{3}$ Division of Advanced Materials Science, Pohang University of Science and Technology (POSTECH), Pohang, Korea Correspondence: Professor MJ Park, Department of Chemistry, Division of Advanced Materials Science, Pohang University of Science and Technology (POSTECH), Room 228, Pohang 790-784, Korea.

E-mail: moonpark@postech.edu

Received 18 November 2015; revised 23 December 2015; accepted 23 December 2015; published online 20 January 2016
} 
in PEO phases. In addition, enhancement of the lithium transport rate by twofold was demonstrated for sulfonic acid-tethered PS-b-PEO. This suggests that the systematic investigation of end-functionalized polymers will increase our knowledge in polymer science, and lead to new insights in advanced battery technologies.

In this report, we describe the synthesis and characterization of block copolymer electrolytes with various end functional groups. Three PS-b-PEO block copolymers with different terminal groups of $-\mathrm{OH},-\mathrm{SO}_{3} \mathrm{H},-\mathrm{COOH}$ were synthesized, while other chemical functionalities remained constant. The introduction of $-\mathrm{COOH}$ group is motivated from the theory, suggesting strong binding affinity of lithium ion with carboxylic acid group, ${ }^{31}$ compared with that with sulfonic acid. A direct comparison of three samples is made to elucidate the relation between embedded terminal groups and lithium ion transport properties for PS-b-PEO electrolytes, based primarily on segregation strength and interfacial properties of the selfassembled morphology. Based on these results, we propose a new platform for improving ion transport efficiency of polymer electrolytes.

\section{EXPERIMENTAL PROCEDURE}

\section{Synthesis of PS-b-PEO-OH, PS-b-PEO-SO ${ }_{3} \mathrm{H}$ and PS-b-PEO-COOH} block copolymers

A set of PS-b-PEO (8.7-11.0 kg mol-1) block copolymers having dissimilar end groups, that is, alcohol $(-\mathrm{OH})$, sulfonic acid $\left(-\mathrm{SO}_{3} \mathrm{H}\right)$ and carboxylic acid $(-\mathrm{COOH})$ were synthesized by the sequential anionic polymerization of styrene and ethylene oxide. To maintain other chemical functionality intact, PS-b-PEO was first prepared in large quantities and divided into three reactors under an Ar-filled glove box, followed by different termination reactions with methanol, 1,3-propanesultone and succinic anhydride for PS-b-PEO-OH, PS-b-PEO-SO ${ }_{3} \mathrm{H}$ and PS-b-PEO-COOH, respectively. Unreacted reagents in the preparation of block copolymers were removed by repeated precipitation and dialysis. The molecular weight and molecular weight distribution of PS-b-PEO-OH were characterized by combining ${ }^{1} \mathrm{H}$ nuclear magnetic resonance $\left({ }^{1} \mathrm{H}-\mathrm{NMR}\right.$, Bruker AVB-300) spectroscopy and gel permeation chromatography (Waters Breeze 2 HPLC). The polydispersity index of PS-b-PEO-OH was 1.08. The completion of termination reactions was confirmed by ${ }^{1} \mathrm{H}-\mathrm{NMR}\left(\mathrm{CDCl}_{3}\right)$ : PS-b-PEO-OH, $\delta$ (p.p.m.) 7.21-6.17 (br m, $420 \mathrm{H},-\mathrm{CH}_{2} \mathrm{CH}\left(\mathrm{C}_{6} \mathrm{H}_{5}\right)-(\mathrm{PS})$ ), 3.91-2.96 (br s, $992 \mathrm{H},-\mathrm{CH}_{2}-\mathrm{CH}_{2}-\mathrm{O}-$ (PEO)), 2.46-0.80 (br m, $249 \mathrm{H},-\mathrm{CH}_{2} \mathrm{CH}\left(\mathrm{C}_{6} \mathrm{H}_{5}\right)-(\mathrm{PS})$ ). PS-b-PEO-SO ${ }_{3} H, \delta$ (p.p.m.) $7.22-6.17$ (br m, $\left.422 \mathrm{H}, \quad-\mathrm{CH}_{2} \mathrm{CH}\left(\mathrm{C}_{6} \mathrm{H}_{5}\right)-(\mathrm{PS})\right), \quad 3.94-2.95$ (br s, $\left.998 \mathrm{H},-\mathrm{CH}_{2}-\mathrm{CH}_{2}-\mathrm{O}-(\mathrm{PEO})\right), 3.46\left(\mathrm{t}, 2 \mathrm{H},-\mathrm{CH}_{2}-\mathrm{O}-\mathrm{CH}_{2}\right.$ $\left.-\mathrm{CH}_{2}-\mathrm{CH}_{2}-\mathrm{SO}_{3} \mathrm{H}\right), 2.66\left(\mathrm{t}, 2 \mathrm{H},-\mathrm{CH}_{2}-\mathrm{O}-\mathrm{CH}_{2}-\mathrm{CH}_{2}-\mathrm{CH}_{2}-\mathrm{SO}_{3} \mathrm{H}\right)$, $2.09\left(\mathrm{~m}, 2 \mathrm{H},-\mathrm{CH}_{2}-\mathrm{O}-\mathrm{CH}_{2}-\mathrm{CH}_{2}-\mathrm{CH}_{2}-\mathrm{SO}_{3} \mathrm{H}\right), 2.44-0.86$ (br m, $251 \mathrm{H}$, $-\mathrm{CH}_{2} \mathrm{CH}\left(\mathrm{C}_{6} \mathrm{H}_{5}\right)-(\mathrm{PS})$ ). PS-b-PEO-COOH, $\delta$ (p.p.m.) 7.12-6.18 (br m, $\left.421 \mathrm{H},-\mathrm{CH}_{2} \mathrm{CH}\left(\mathrm{C}_{6} \mathrm{H}_{5}\right)-(\mathrm{PS})\right), 4.23\left(\mathrm{t}, 2 \mathrm{H},-\mathrm{CH}_{2}-\mathrm{OCO}-\mathrm{CH}_{2}-\mathrm{CH}_{2}\right.$ $-\mathrm{CO}_{2} \mathrm{H}$ (PEO)), 3.94-2.95 (br s, $996 \mathrm{H},-\mathrm{CH}_{2}-\mathrm{CH}_{2}-\mathrm{O}-(\mathrm{PEO})$ ), 2.63 $\left(\mathrm{m}, 4 \mathrm{H},-\mathrm{CH}_{2}-\mathrm{OCO}-\mathrm{CH}_{2}-\mathrm{CH}_{2}-\mathrm{CO}_{2} \mathrm{H}\right), 2.39-0.87$ (br $\mathrm{m}, 252 \mathrm{H}$, $\left.-\mathrm{CH}_{2} \mathrm{CH}\left(\mathrm{C}_{6} \mathrm{H}_{5}\right)-(\mathrm{PS})\right)$.

\section{Synthesis of MPEO-SO ${ }_{3} \mathrm{H}$ and MPEO-COOH homopolymers}

A polyethylene glycol methyl ether (MPEG, $10 \mathrm{~kg} \mathrm{~mol}^{-1}$ ) was purchased from Sigma-Aldrich (Yongin-si, Gyeonggi-do, Korea) and was used as received. $1 \mathrm{~g}$ of MPEG $(0.1 \mathrm{mmol})$ was dissolved in $100 \mathrm{ml}$ of benzene and $25 \mathrm{mg}$ of sodium hydride ( $1 \mathrm{mmol}$ ) was added into the mixtures. After stirring for $12 \mathrm{~h}, 250 \mathrm{mg}$ of 1,3-propanesultone $(2 \mathrm{mmol})$ or $200 \mathrm{mg}$ of succinic anhydride $(2 \mathrm{mmol})$ was added to the reaction mixtures to substitute terminal alcohol of the MPEG by $-\mathrm{SO}_{3} \mathrm{H}$ or $-\mathrm{COOH}$ group, respectively. After precipitation in ether, polymers were dissolved in methanol and 20 eq. excess amount of methanol-diluted $\mathrm{HCl}$ was added. The resultant MPEOS and MPEOC polymers were precipitated in ether three times and recovered by vacuum drying at room temperature for 2 days and at $50{ }^{\circ} \mathrm{C}$ for 5 days. The exchange efficiency was determined as $>95 \%$ by ${ }^{1} \mathrm{H}-\mathrm{NMR}$ spectroscopy.

\section{Preparation of lithium salt-doped polymers}

The polymers and pre-determined amounts of bis(trifluoromethane) sulfonimide lithium salt (LiTFSI) were weighed into glass vials, and $\sim 1 \mathrm{wt} \%$ solutions were prepared using benzene and methanol mixtures (40/60 vol\%). Solution was stirred overnight at room temperature and the LiTFSI-doped polymers were solvent cast under an $\mathrm{Ar}$ atmosphere, followed by vacuum drying at $70^{\circ} \mathrm{C}$ for 7 days. To exclude the issue of water contamination of hygroscopic samples, all sample preparations were performed under an Ar-filled glove box with oxygen and moisture levels below 0.1 p.p.m.

\section{Small angle $\mathrm{X}$-ray scattering}

The neat and LiTFSI-doped block copolymers were laminated into an airtight sample cell within an Ar-filled glove box to avoid the issue of water contamination, followed by thermal annealing at $120^{\circ} \mathrm{C}$ for 7 days. Synchrotron small-angle X-ray scattering (SAXS) measurements on these samples were performed using the 3C SAXS beam line at the Pohang Light Source (PLS-II). Sample temperature was controlled within $\pm 0.2^{\circ} \mathrm{C}$ using a sample stage provided by the PLS-II. The wavelength $(\lambda)$ of the incident X-ray beam was $0.118 \mathrm{~nm}\left(\Delta \lambda / \lambda=10^{-4}\right)$, and sample-to-detector distance of $2 \mathrm{~m}$ was used yielding scattering wave vector $q(q=4 \pi \sin (\theta / 2) / \lambda$, where $\theta$ is the scattering angle) in the range $0.1-2.0 \mathrm{~nm}^{-1}$. The resulting two-dimensional scattering data were averaged azimuthally to obtain intensity versus $q$.

\section{Transmission electron microscopy}

The neat and LiTFSI-doped block copolymers prepared for SAXS experiments were cryo-microtomed at $-120^{\circ} \mathrm{C}$ to obtain thin sections with thicknesses in the 80-120 nm range using a RMC Boeckeler PT XL Ultramicrotome (Tucson, AZ, USA). The electron contrast in the samples was enhanced by exposure to ruthenium tetroxide $\left(\mathrm{RuO}_{4}\right)$ vapor for $30 \mathrm{~min}$. Imaging of stained samples was performed with a Hitachi H-800 microscope (Tokyo, Japan) operating at $80 \mathrm{kV}$

\section{Conductivity measurements}

In an Ar-filled glove box, the through-plane conductivities of Li-salt-doped polymer membranes were measured using a home-built two-electrode cell consisting of $1.25 \times 1.25 \mathrm{~cm}$ stainless-steel blocking electrodes and $1 \times 1 \mathrm{~cm}$ Pt working/counter electrodes. Data were collected using a VersaSTAT 3 (Princeton Applied Research, Oak Ridge, TN, USA) impedance analyzer operating over a frequency range of $1 \sim 100000 \mathrm{~Hz}$.

\section{Elucidation of intermolecular interactions in end-functionalized PEO}

The melting transition of each sample was measured by differential scanning calorimetry (DSC) using model Q20 (TA Instruments, New Castle, DE, USA). The samples were encapsulated in aluminum pans within glove box prior to the measurements to exclude the issue of water contamination of hygroscopic samples. DSC thermograms of all samples were obtained at a fixed heating and cooling scan rate of $10^{\circ} \mathrm{C} \mathrm{min}-1$. X-ray power diffraction (XRD) experiments were carried out using Rigaku D/MAX-2500 (Tokyo, Japan) (CuKo, $\lambda=1.54 \AA$ ) in $2 \theta$ range of $10-40^{\circ}$. Fourier transformed infrared (FT-IR) spectra of the samples were recorded at room temperature using a Spectrum Two IR spectrometer (PerkinElmer, Waltham, MA, USA) where 32 accumulations were signal-averaged at a resolution of $1 \mathrm{~cm}^{-1}$.

\section{RESULTS AND DISCUSSION}

Synthesis and morphology of end-functionalized PS-b-PEO block copolymers

The PS-b-PEO-OH, PS-b-PEO-SO ${ }_{3} \mathrm{H}$ and PS-b-PEO-COOH block copolymers, having dissimilar end groups, were obtained by different termination reactions of anionic polymerization with methanol, 1,3-propanesultone and succinic anhydride, respectively. The synthetic procedures and molecular structures of resultant block copolymers are shown in Figure 1a. Hereafter, SEO refers to PS-b-PEO-OH, while the two other copolymers possessing $-\mathrm{SO}_{3} \mathrm{H}$ and $-\mathrm{COOH}$ terminal 

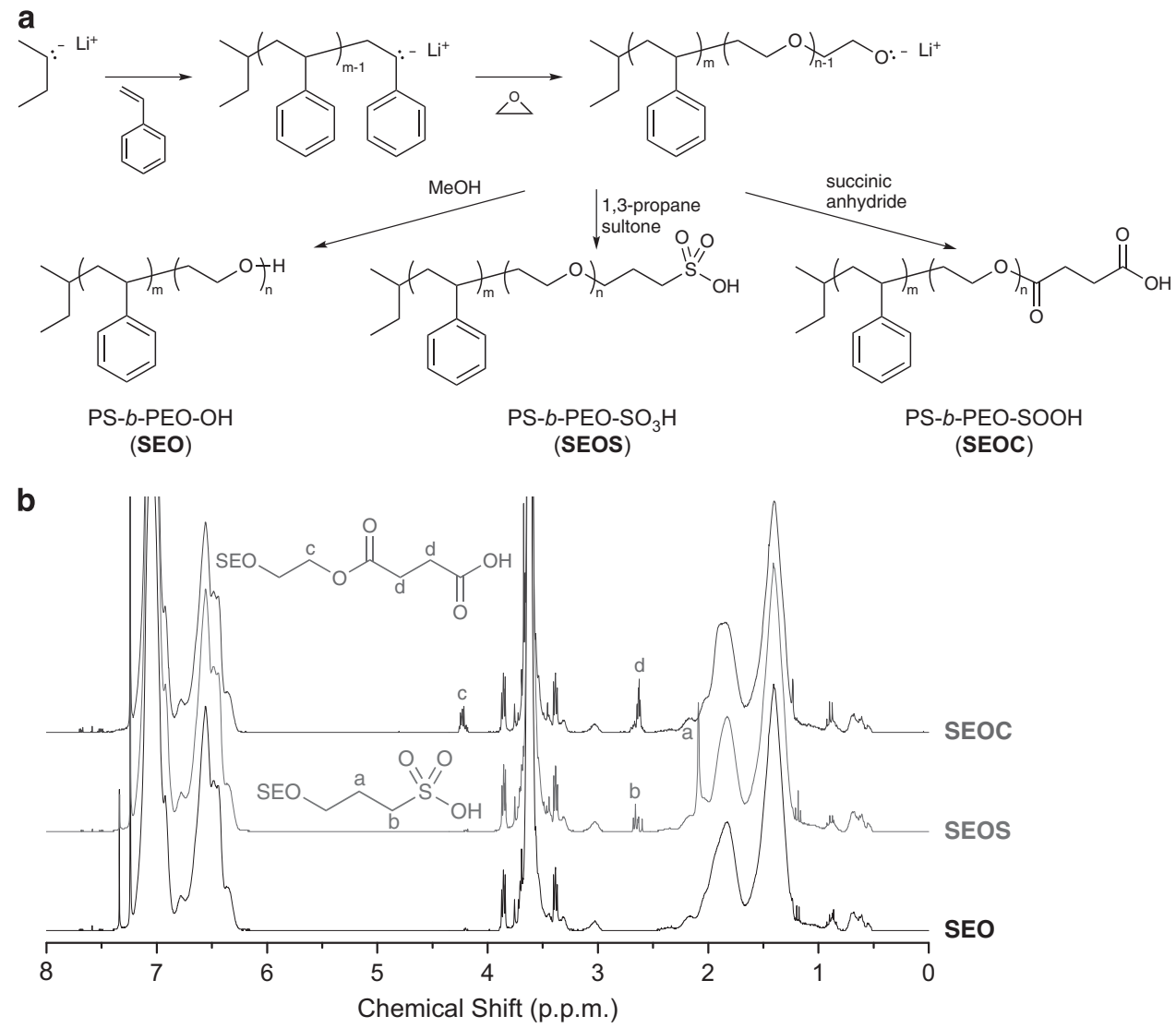

Figure 1 (a) Synthetic procedure of PS-b-PEO block copolymers having dissimilar end groups of alcohol $(-\mathrm{OH})$, sulfonic acid $\left(-\mathrm{SO}_{3} \mathrm{H}\right)$ and carboxylic acid $(-\mathrm{COOH})$ via termination steps of anionic polymerization. (b) ${ }^{1} \mathrm{H}-\mathrm{NMR}$ spectra of SEO, SEOS and SEOC; the characteristic peaks of SEOS and SEOC are assigned in the figure. NMR, nuclear magnetic resonance. A full color version of this figure is available at Polymer Journal online.

groups are referred to as SEOS and SEOC, respectively. Note that the molecular weight of precursor SEO was determined as $8.7-11.0 \mathrm{~kg} \mathrm{~mol}^{-1}$ and the molar mass increments of end-modified PEO in SEOS and SEOC were as small as $0.12 \mathrm{~kg} \mathrm{~mol}^{-1}$. This small difference will allow a straightforward investigation of the samples to unravel the effects of terminal groups on the morphology and ion transport properties of PS-b-PEO block copolymers.

Figure $1 \mathrm{~b}$ shows ${ }^{1} \mathrm{H}-\mathrm{NMR}$ spectra $\left(\mathrm{CDCl}_{3}\right)$ of SEO, SEOS and SEOC copolymers. For SEOS, having a $-\mathrm{SO}_{3} \mathrm{H}$ end group, the triplet at 2.66 p.p.m. and singlet at 2.09 p.p.m. appeared as a result of the tethered 1,3-propanesultone, as shown in the figure. The attachment of a $-\mathrm{COOH}$ group to SEO (that is, SEOC) resulted in the appearance of new peaks at 4.23 and 2.63 p.p.m. The ${ }^{1} \mathrm{H}-\mathrm{NMR}$ spectra confirmed $>95 \%$ acid substitution on the PEO chains for both SEOS and SEOC.

The morphologies of SEO, SEOS, SEOC block copolymers were examined by SAXS experiments. Representative SAXS profiles measured at $25^{\circ} \mathrm{C}$ are shown in Figure 2a, which were unchanged in the temperature window of interest. The SEO having a $-\mathrm{OH}$ terminal group showed poorly defined morphology with a domain spacing of $15.9 \mathrm{~nm}$. The inclusion of terminal acid units in SEO caused the development of an ordered lamellar (LAM) structure for both SEOS and SEOC, accompanied by substantial reductions in full width at half maximum (fwhm) of the primary peak, and significant increases in domain size to 17.1 (SEOS) and $17.2 \mathrm{~nm}$ (SEOC), as plotted in the inset of Figure 2a. This suggests that the incorporation of terminal acid groups onto PEO in PS-b-PEO resulted in increased segregation strength, as described by the effective Flory-
Huggins interaction parameter, $\chi_{\text {eff }}$, between acid-tethered PEO and PS phases, to stabilize the ordered phase. We also infer that acid moieties increased the free volume of PEO phases, thereby causing the increase in domain size. This observation is intriguing, given that the end group concentration of PEO in PS-b-PEO employed in this study is as small as $0.4 \mathrm{~mol} \%$.

The self-assembly behavior of SEO, SEOS and SEOC copolymers was further investigated by doping with lithium salt (LiTFSI, TFSI $\left.=\left[\left(\mathrm{CF}_{3} \mathrm{SO}_{2}\right)_{2} \mathrm{~N}\right]^{-}\right)$. The resultant SAXS profiles at $r=0.03$ $\left(r=\left[\mathrm{Li}^{+}\right] /[\mathrm{EO}]\right)$ are shown in Figure $2 \mathrm{~b}$, and all three samples display LAM morphologies. This indicates the occurrence of a disorder-toorder transition for the SEO copolymers as a result of LiTFSI doping, which we attribute to the increased $\chi_{\text {eff }}$ between the PEO/LiTFSI and PS phases. This is in good agreement with the body of literature on PS-b-PEO block copolymer electrolytes. ${ }^{32}$ Again, stronger Bragg peaks and narrower fwhm of polymers carrying acidic terminal groups were observed as compared with those of $-\mathrm{OH}$ terminated SEO.

As plotted in the inset of Figure 2b, the domain size of SEOS became the largest with LiTFSI doping, with decreasing size from SEOS $(19.9 \mathrm{~nm})$, to SEOC $(19.5 \mathrm{~nm})$ and lastly SEO $(19.1 \mathrm{~nm})$. This result is not due to a simple increase in the $\chi_{\text {eff }}$ value by the addition of LiTFSI because the strongest microphase separation was seen for SEOC. We hypothesized that the LiTFSI doping induced additional Coulombic interactions between $\mathrm{Li}^{+}$and the terminal groups, which afforded dissimilar degrees of swelling of PEO phases, depending on the type of acid unit. This speculation is further supported in the following sections. 

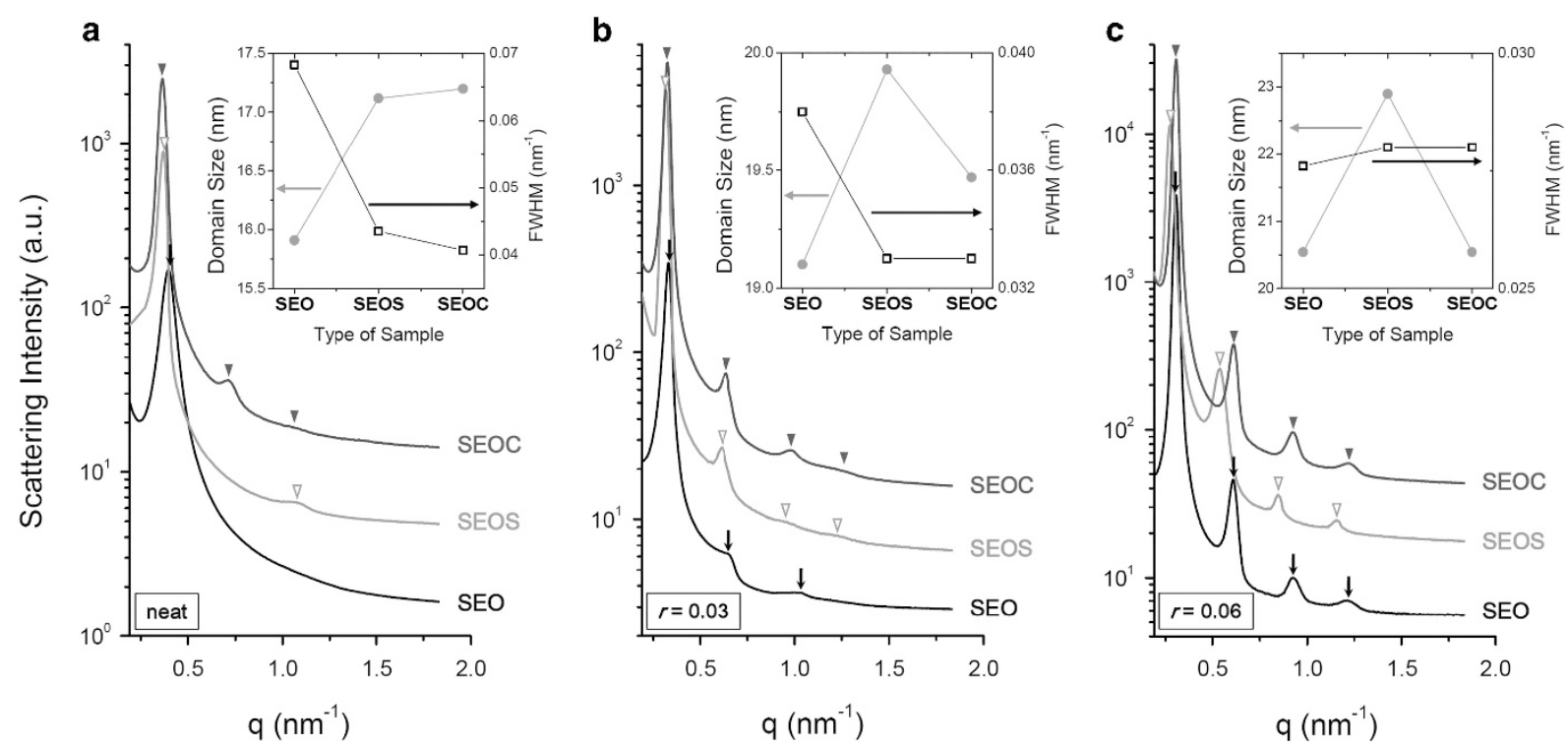

Figure 2 SAXS profiles of (a) neat SEO, SEOS and SEOC block copolymers and (b and $\mathbf{c}$ ) LiTFSI-doped samples at $r=0.03$ and at $r=0.06$, measured at $25^{\circ} \mathrm{C}$. The inverted symbols in the figure indicate Bragg peaks at the ratio of 1:2:3:4. The domain size and fwhm of primary peak of each sample are plotted in the insets. SAXS, small-angle X-ray scattering. A full color version of this figure is available at Polymer Journal online.

Note that the LAM structure is increasingly well-organized as $r$ increases in value up to 0.09 . Figure $2 \mathrm{c}$ shows representative SAXS data obtained with $r=0.06$, where the increased domain sizes of 20.5, 22.9 and $20.5 \mathrm{~nm}$ were revealed for SEO, SEOS and SEOC samples, respectively. Again, the largest domain size was identified for SEOS. This confirms that while the PEO, PEOS and PEOC phases ultimately swell in the presence of LiTFSI by plasticizing effects (with reduced PEO crystallinity), ${ }^{32,33}$ the degree of swelling is linked to the type of terminal group of PEO. We will discuss the origin for these results in the following sections, based primarily on dissimilar degrees of salt dissociation and different chain conformations of end-functionalized polymers. Interestingly, essentially no difference in fwhm is seen for SEO, SEOS and SEOC samples at $r=0.06$. This leads us to conclude that at increased LiTFSI-loading, an ample amount of $\mathrm{Li}^{+}$ions and TFSI ${ }^{-}$counter ions were free to distribute along the ether backbone. Thus, the thermodynamics is dominated by the ionic interactions of embedded salt over the intermolecular interactions of terminal acid group.

Transmission electron microscopy images in Figure 3 illustrate the morphology of PS-b-PEO block copolymers associated with the endfunctional groups, where a representative set of images obtained with a small degree of LiTFSI doping $(r=0.03)$ is presented. Defective LAM morphology was revealed for the $-\mathrm{OH}$ terminated SEO, whereas the inclusion of a single $-\mathrm{SO}_{3} \mathrm{H}$ or $-\mathrm{COOH}$ terminal group to the SEO resulted in the development of more well-defined LAM structures. This is in good agreement with the SAXS results shown in Figure $2 \mathrm{~b}$.

\section{Terminal-group-driven intermolecular interactions in PEO phases} We performed XRD experiments to investigate changes in crystallinity of PEO chains in relation to end functionalization. Figure 4a shows XRD patterns of neat SEO, SEOS and SEOC block copolymers. The diffraction peaks at $2 \theta$ of $19.7^{\circ}$ and $23.9^{\circ}$, marked with inverted filled triangles $(\boldsymbol{\nabla})$, indicate typical XRD patterns of crystalline PEO. The XRD patterns of SEOS and SEOC clearly indicated reduction in the degree of crystallinity of PEO on the attachment of $-\mathrm{SO}_{3} \mathrm{H}$ and $-\mathrm{COOH}$ groups, and the reduction was more apparent for SEOC.
The melting transition temperature $\left(T_{\mathrm{m}}\right)$ of each sample was measured by DSC (DSC), and the results are shown in Figure $4 \mathrm{~b}$. The DSC thermograms of SEOS and SEOC displayed reduced heat of melting at lower melting temperatures than that of SEO, with $\Delta H_{\mathrm{m}}=66.5,61.6,56.4 \mathrm{~J} \mathrm{~g}^{-1}$ and $T_{\mathrm{m}}=55.5,53.1,51.1^{\circ} \mathrm{C}$ for $\mathrm{SEO}$, SEOS and SEOC, respectively. More significant reduction in the degree of crystallinity of PEO was again seen in SEOC, consistent with the XRD patterns in Figure 4a. This suggests that the intermolecular interactions of acid-terminated PEO chains affect the packing density of PEO chains, which is intimately associated with the increased domain sizes of SEOS and SEOC block copolymers, as shown in Figure 1a.

To determine the types of intermolecular interactions in PEO caused by terminal group substitution, FT-IR spectra of SEO, SEOS and SEOC block copolymers were recorded. Figure 5 shows the FT-IR spectra acquired at room temperature in the wavenumber region of 3900-1050 $\mathrm{cm}^{-1}$. In the absence of lithium salt, shown in Figure 5a, the IR peak intensities corresponding to stretching of $\mathrm{C}-\mathrm{O}-\mathrm{C}$ (the PEO backbone) were found to be reduced and shifted for SEOC, indicating that the $-\mathrm{COOH}$ terminal group participated in hydrogenbonding interactions with ether oxygen atoms. Unlike SEOC, no significant change in $\nu_{\mathrm{C}-\mathrm{O}-\mathrm{C}}$ was seen for SEOS, but instead strong $-\mathrm{OH}$ peak signals were observed in the $3700-3100 \mathrm{~cm}^{-1}$ region. This implies that much of the $-\mathrm{SO}_{3} \mathrm{H}$ takes part in the quadrupole formation.

Note in passing that the balance of dimer formation by the chain ends and hydrogen-bonding interactions between - $\mathrm{COOH}$ group and ether oxygen of PEO chains can be controlled by changing molecular weight of PEOC (that is, end group concentration). In other words, the higher molecular weight of PEOC resulted in more significant hydrogen-bonding interactions by lowering the chance of dimerization. This was confirmed by monitoring the $\nu \mathrm{CO}$ peak in range of $1740-1710 \mathrm{~cm}^{-1}, 34,35$ where the reduction in PEOC molecular weight resulted in the shift of $\nu \mathrm{CO}$ peak to lower wavenumber (data not shown here).

The FT-IR spectra of SEO, SEOS and SEOC with a small degree of LiTFSI doping $(r=0.03)$, compared with neat samples, are also shown 


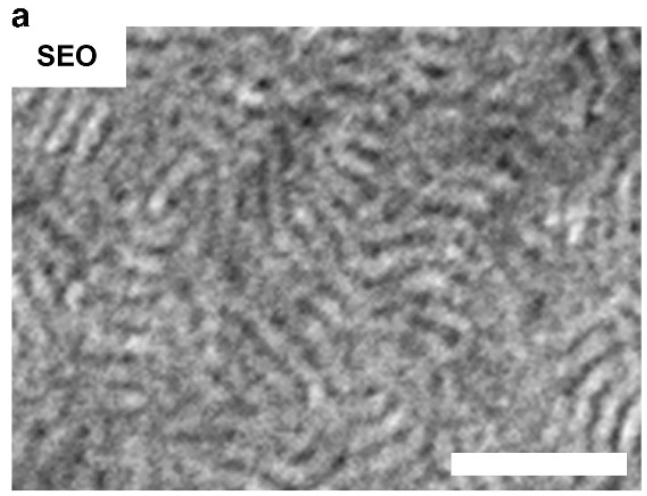

b

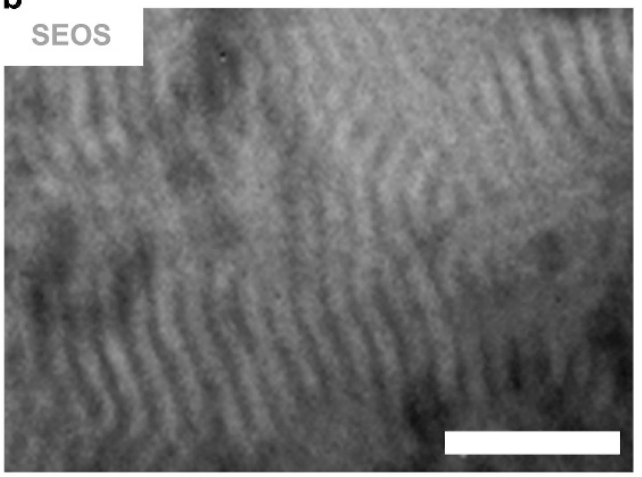

C

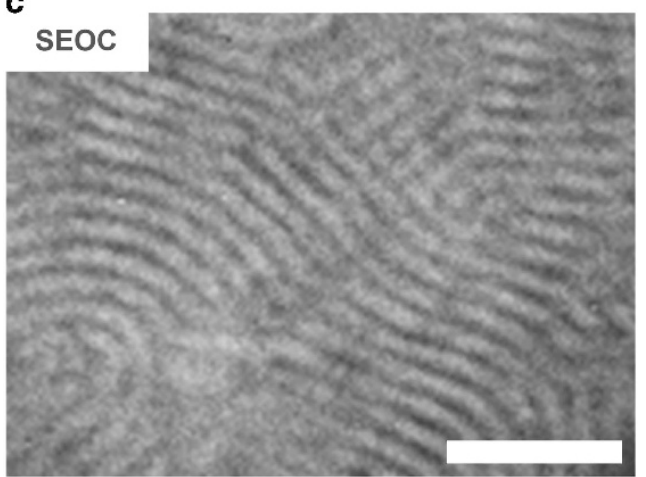

Figure 3 Transmission electron micrographs of (a) SEO, (b) SEOS and (c) SEOC block copolymers obtained with LiTFSI doping $(r=0.03)$, representing dissimilar strength of segregation associated with end-functional groups. PEO phases were darkened by $\mathrm{RuO}_{4}$ staining, and scale bars represent $100 \mathrm{~nm}$. A full color version of this figure is available at Polymer Journal online.

in Figure 5b. These spectra provide further indications of dissimilar intermolecular interactions, depending on the terminal group. As assigned in the figure, increased IR intensities of the characteristic peaks of $\mathrm{TFSI}^{-}$anions were evident on the replacement of $-\mathrm{OH}$ by $\mathrm{SO}_{3} \mathrm{H}$ or $-\mathrm{COOH}$. This denotes the dissociation of LiTFSI for SEOC and SEOS facilitated by the attractive Coulombic interactions between $\mathrm{Li}^{+}$-ion and terminal acid groups, as proposed in Figure $2 \mathrm{~b}$.

Based on the results obtained thus far, Figure 6 depicts the proposed inter-chain interactions of PEO phases in the SEO, SEOS and SEOC samples. For neat SEOS and SEOC copolymers, the terminal acid units of PEO chains could contribute to hydrogen-bonding interactions and quadrupole interactions with neighboring chains. The tendency to form quadrupoles seems to be dissimilar between $-\mathrm{SO}_{3} \mathrm{H}$ and $-\mathrm{COOH}$, leading to different intermolecular arrangements. We
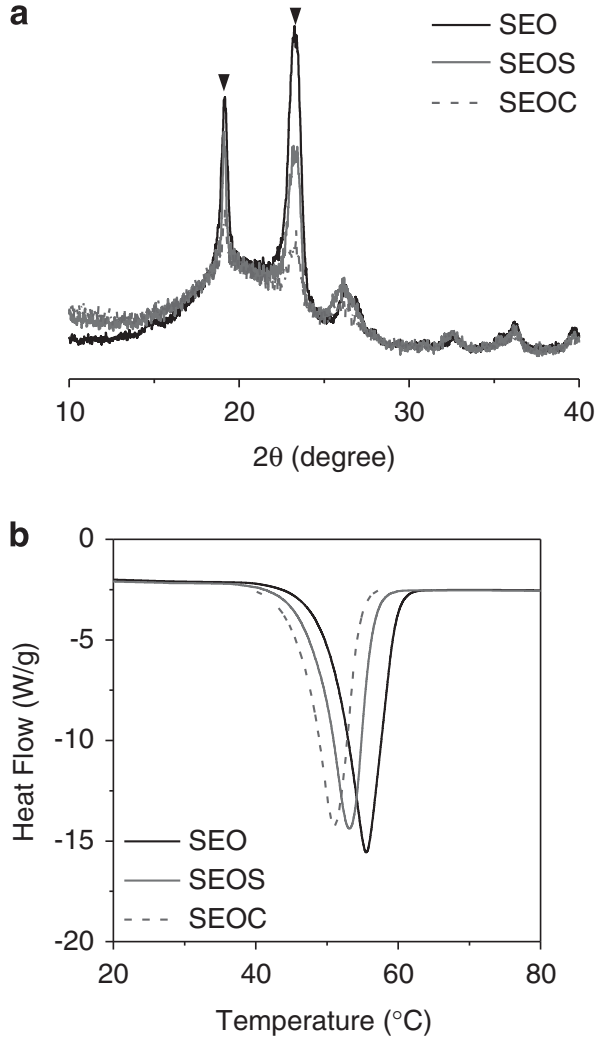

Figure 4 (a) XRD patterns and (b) DSC thermograms of neat SEO, SEOS and SEOC block copolymers. The inverted filled triangles $(\boldsymbol{\nabla})$ in a show typical diffraction peaks of crystalline PEO $\left(2 \theta=19.7^{\circ}, 23.9^{\circ}\right)$. DSC, differential scanning calorimetry; XRD, X-ray powder diffraction. A full color version of this figure is available at Polymer Journal online.

speculate that such intermolecular interactions lead to the reduction in compatibility of PEO with PS phases, thereby influencing the interfacial properties of self-assembled PS-b-PEO. On the addition of lithium salt, the $\mathrm{Li}^{+}$ion coordinates with ether oxygen atoms for all three samples, but it additionally contributes to attractive electrostatic interactions with terminal acid units, yielding differential swelling of PEO phases as a function of the terminal functional group.

\section{Ion transport properties of PS-b-PEO having different terminal groups}

The preparation of high performance polymer electrolytes is essential for their practical use in lithium batteries. To this end, establishment of a new methodology for the design of efficient ion-conducting polymers is of importance. We investigated the effect of terminal groups of $\mathrm{PEO}$ in PS-b-PEO on ion transport properties in PS-b-PEO/ LiTFSI complexes. Figure 7 a show two sets of conductivity data, obtained using AC impedance spectroscopy at fixed $\mathrm{Li}^{+}$ion concentrations of $r=0.03$ and 0.06 , respectively. Overall, the conductivity, in decreasing order, has $\mathrm{SEO}>\mathrm{SEOS}>\mathrm{SEOC}$ regardless of the salt concentration. This is ascribed to the slow segmental motion of acid-tethered PEO chains by forming internal ion-dipole and dipole-dipole interactions, which are tied to the low conductivity. Increasing LiTFSI concentration from $r=0.03$ and 0.06 led to three to fourfold increased enhanced conductivity for the samples. Solid lines in the figures were obtained by Vogel-Tammann-Fulcher (VTF) analysis. The obtained potential barriers to ion conduction were 693 


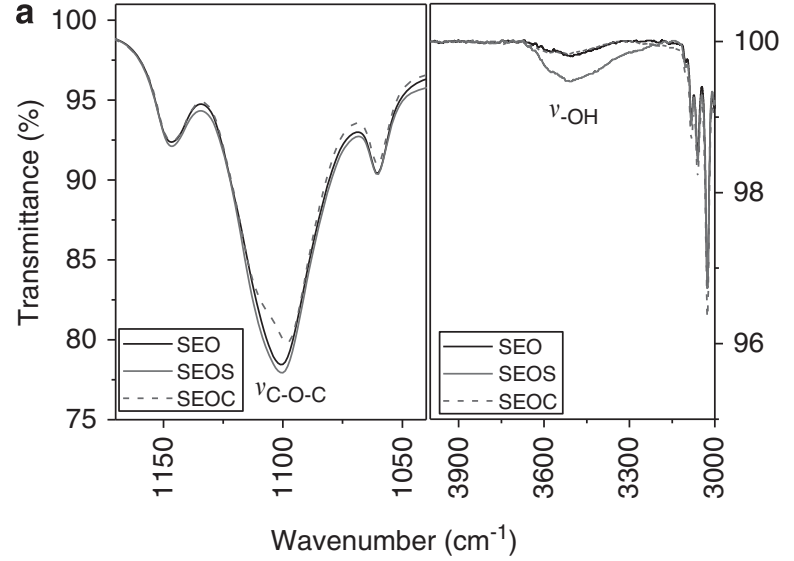

b

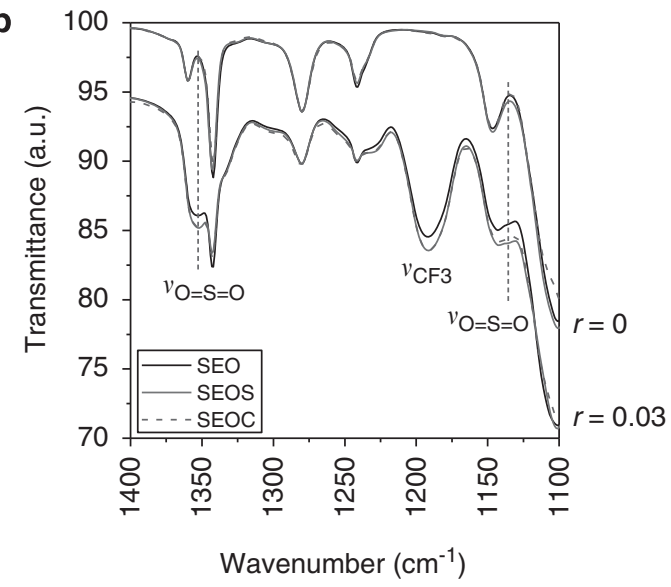

Figure 5 FT-IR spectra of (a) neat SEO, SEOS and SEOC block copolymers indicating intermolecular hydrogen-bonding interactions and (b) SEO, SEOS and SEOC with a small degree of LiTFSI doping $(r=0.03)$ as compared with those of neat samples, illuminating enhanced dissociation of $\mathrm{TFSI}^{-}$anions in end-functionalized samples. Relevant peaks in the wavenumber range 3900$1050 \mathrm{~cm}^{-1}$ are assigned in the figure. FT-IR, Fourier transform infrared. A full color version of this figure is available at Polymer Journal online.
$\mathrm{K}, 708 \mathrm{~K}, 715 \mathrm{~K}$ (at $r=0.03$ ) and $745 \mathrm{~K}, 758 \mathrm{~K}, 803 \mathrm{~K}$ (at $r=0.06$ ) for SEO, SEOS and SEOC, respectively.

Similar tendency in ionic conductivity was confirmed for MPEO, MPEOS and MPEOC homopolymers, as shown in Figure 7b. In a temperature window of $45-90{ }^{\circ} \mathrm{C}$ and at $\mathrm{Li}^{+}$concentrations of $r=0.03$ and 0.06 , more than $50 \%$ higher conductivity was observed for MPEO with $-\mathrm{OH}$ terminal groups, compared with those of MPEOS and MPEOC. This indicates that the ion mobility with end-modified MPEO chains is influenced largely by the type of end group, which changes chain dynamics via strong intermolecular interactions. Based on the lowest conductivity seen for $-\mathrm{COOH}$ terminated samples, we infer that the strongest intermolecular associations occurred in MPEOC phases. The activation barriers to ion conduction determined by the VTF analysis were $620 \mathrm{~K}, 611 \mathrm{~K}, 645 \mathrm{~K}$ (at $r=0.03$ ) and $653 \mathrm{~K}$, $654 \mathrm{~K}, 712 \mathrm{~K}$ (at $r=0.06$ ) for MPEO, MPEOS and MPEOC, respectively.

To exclude the effects of chain segmental motion elucidate in the relation between morphology and ionic conductivity of these PS-b-PEO block copolymers with different terminal groups, the conductivity data shown in Figure $7 \mathrm{a}$ were normalized based on the data in Figure 7b, respectively. We employed equation (1) given below, developed by Sax and Ottino: ${ }^{36}$

$$
\sigma_{\text {nor }}=\frac{\sigma_{\text {block }}}{\phi_{\text {cond }} \sigma_{\text {homo }}}
$$

where $\sigma_{\text {nor }}$ is the normalized conductivity, $\sigma_{\text {block }}$ is the conductivity of the salt-doped block copolymers, $\sigma_{\text {homo }}$ is that of the homopolymer analogs at a given Li-salt concentration, and $\phi_{\text {cond }}$ is the volume fraction of conducting phases in block copolymers. A fixed $\phi_{\text {cond }}$ value of 0.558 , calculated from the density and molecular weight of PS and neat $\mathrm{PEO}$, was used in the relation.

Figure $7 \mathrm{c}$ show the $\sigma_{\text {nor }}$ for SEO, SEOS and SEOC block copolymers at $\mathrm{Li}^{+}$concentrations of $r=0.03$ and 0.06 , respectively. In a temperature window of $40-90^{\circ} \mathrm{C}$, the $\sigma_{\text {nor }}$ values of SEOC were qualitatively similar to those of SEOS at $r=0.03$, as shown in Figure 7c. However, the value noticeably surpassed that of SEO at the same salt concentration. The substantially augmented $\mathrm{Li}^{+}$transport efficiency

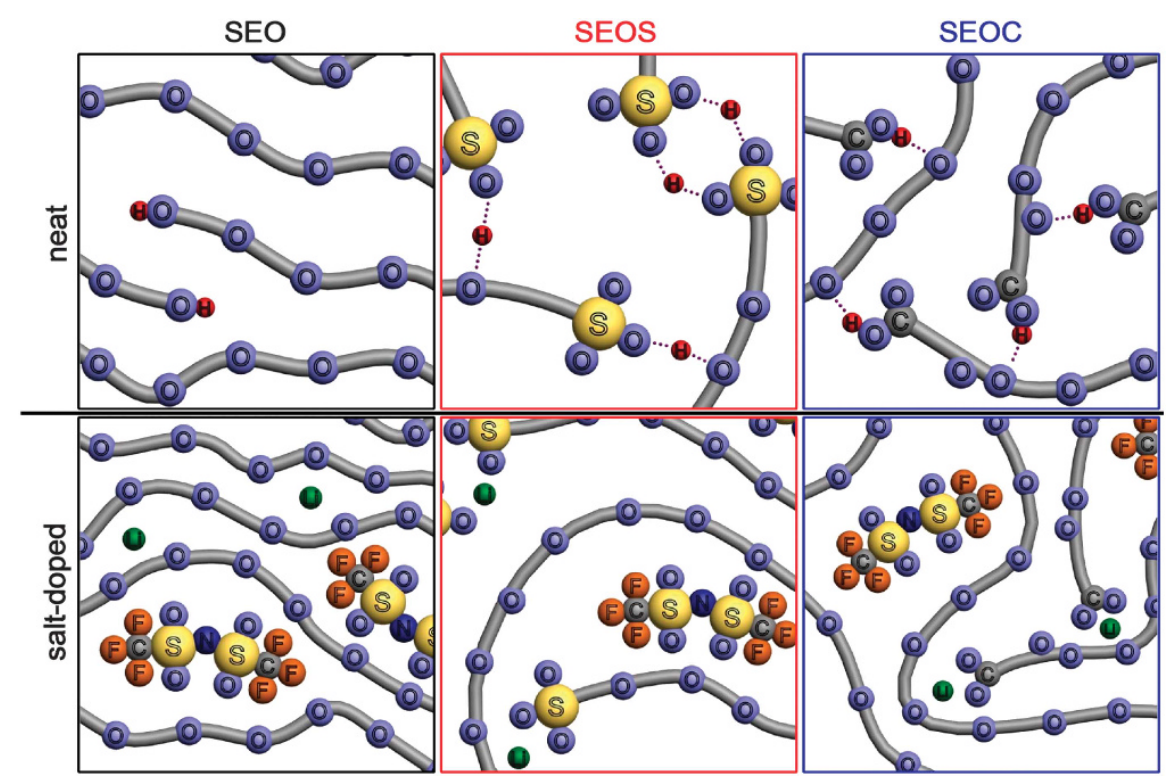

Figure 6 Schematic drawings of the proposed intermolecular interactions of PEO, PEOS and PEOC in PS-b-PEO block copolymers in the absence and presence of lithium salt. 
a

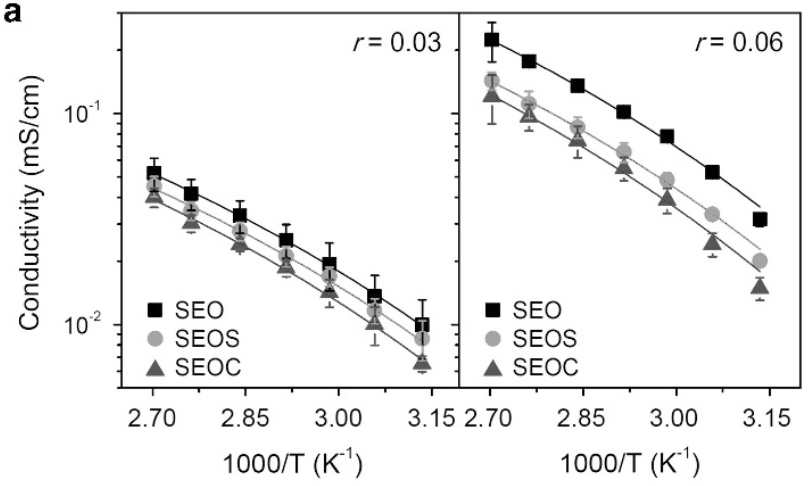

b
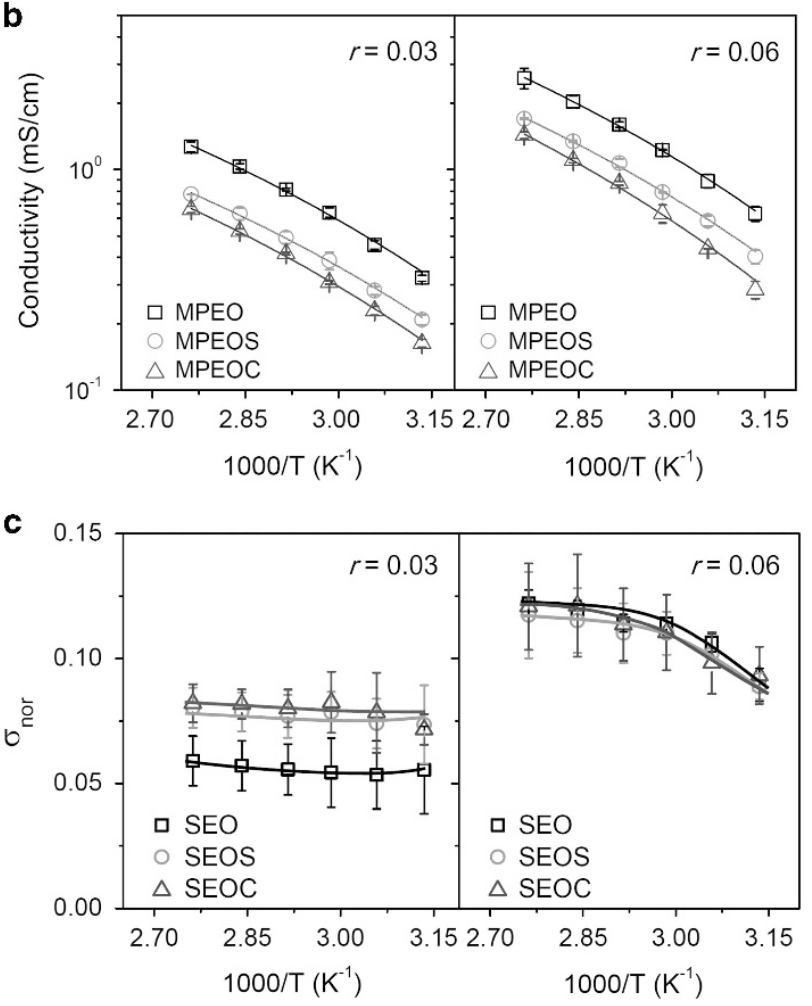

Figure 7 Temperature-dependent ionic conductivity of (a) SEO, SEOS and SEOC block copolymers (filled symbols) and (b) MPEO, MPEOS and MPEOC homopolymers (open symbols) at $r=0.03$ and $r=0.06$. Solid lines in the figures were obtained by VTF analysis. (c) Normalized conductivity $\left(\sigma_{\text {nor }}\right)$ of SEO, SEOS and SEOC at $r=0.03$ and $r=0.06$. Solid lines in $\mathbf{c}$ show the best regression of data. A full color version of this figure is available at Polymer Journal online.

across the acid-tethered PEO domains is closely related to the structural advantages of well-defined LAMs with sharp interfaces, enabling less tortuous ion conduction pathways with low levels of interfacial mixing with the PS phases. In contrast, at $r=0.06$ (Figure $7 \mathrm{c}$ ), the $\sigma_{\text {nor }}$ values of all three samples were similar over the entire range of temperature, presumably due to the analogous segregation strength, as shown in Figure $2 \mathrm{c}$. The high $\sigma_{\text {nor }}$ values at high salt concentration is also interesting to note. We therefore concluded that the terminal group-driven modulation of interfaces of self-assembled structures has a profound impact on the respective ion transport efficiencies across ordered grains.

In our previous study ${ }^{30}$ we used low molecular weight PS-b-PEO $\left(6.6-4.6 \mathrm{~kg} \mathrm{~mol}^{-1}\right)$ to investigate the effects of end-functional groups on morphology and conductivity. We anticipated that the impact of end-functional groups would be weakened as the molecular weight of PEO increased, due to the decreased end group concentration. However, contrary to our prediction, the terminal group-driven modulation of morphology and ion transport properties was still discernible for PS-b-PEO with more than twice the molecular weight of PEO, which is noteworthy.

Our results in this study present new avenues for designing efficient polymer electrolytes through simple chemistry, which are applicable to various emerging electrochemical devices, and energy storage and conversion technologies. Although the absolute conductivity values were low for acid-tethered polymers, understanding of the relation between interfaces and ion transport efficiency for self-assembled polymer electrolytes established in the present study is fundamentally important for polymer science. The low conductivity may be resolved by preparing a new set of end-modified polymers with relatively bulky acidic moieties, which is currently undergoing in our laboratory. The development of three-dimensional morphologies having co-continuous conducting phases from these polymers is also endeavoring to improve the transport efficiency.

\section{CONCLUSIONS}

We investigated the route to control morphology and ion transport properties of PS-b-PEO block copolymers by the inclusion of a single end-functional group. Stronger Bragg peaks and narrower interfaces were observed in block polymers carrying acidic terminal groups, in accordance with significant changes in domain size as compared with those of $-\mathrm{OH}$ terminated samples. This was attributed to modulated intermolecular interactions in acid-tethered PEO phases and lowered compatibility of functionalized PEO with PS phases. The relation of embedded terminal groups and ion transport properties for PS-b-PEO electrolytes was further elucidated. The slow segmental motion of acid-tethered PEO chains was related to the low conductivity of PS-b$\mathrm{PEO}$, and the normalized conductivity of acid-terminated PS-b-PEO noticeably surpassed that of PS-b-PEO with -OH end groups at a low degree of salt loading. The substantially augmented $\mathrm{Li}^{+}$transport efficiency across the end-functionalized PEO domains was intimately associated with the structural advantages of sharp interfaces, with low levels of interfacial mixing with PS phases. Given that the PEO phases ultimately swell in the presence of excessive lithium salt, the differential effects of terminal groups on the interfaces and ion transport efficiency of PS-b-PEO vanished at high level of salt concentration. This work presents new fundamental experimental insights for the future design of polymer electrolytes.

\section{CONFLICT OF INTEREST}

The authors declare no conflict of interest.

\section{ACKNOWLEDGEMENTS}

This work was financially supported by the Global Frontier R\&D program on Center for Multiscale Energy System funded by the National Research Foundation under the Ministry of Science, ICT \& Future, Korea.

1 Murata, K., Izuchi, S. \& Yoshihisa, Y. An overview of the research and development of solid polymer electrolyte batteries. Electrochim. Acta 45, 1501-1508 (2000).

2 Tarascon, J.-M. \& Armand, M. Issues and challenges facing rechargeable lithium batteries. Nature 414, 359-367 (2001).

3 Xu, K., Ding, M. S., Zhang, S., Allen, J. L. \& Jow, T. R. An attempt to formulate nonflammable lithium ion electrolytes with alkyl phosphates and phosphazenes. J. Electrochem. Soc. 149, A622-A626 (2002). 
4 Abe, K., Yoshitake, H., Kitakura, T., Hattori, T., Wang, H. \& Yoshio, M. Additivescontaining functional electrolytes for suppressing electrolyte decomposition in lithiumion batteries. Electrochimica Acta 49, 4613-4622 (2004).

5 Quartarone, E., Mustarelli, P. \& Magistris, A. PEO-based composite polymer electrolytes. Solid State Ionics 110, 1-14 (1998).

6 Kim, D.-W., Park, J.-K., Rhee, H.-W. \& Kim, H.-D. Ionic conductivity and ${ }^{7} \mathrm{Li}$ NMR study of solid polymer electrolytes based on polyetherurethane copolymer networks. Polymer J. 26, 993-1001 (1994).

7 Rosso, M., Brissot, C., Teyssot, A., Dolle, M., Sannier, L., Tarascon, J. M., Bouchetc, R. \& Lascaud, S. Dendrite short-circuit and fuse effect on Li/polymer/Li cells. Electrochim. Acta 51, 5334-5340 (2006).

8 Harry, K. J., Hallinan, D. T., Parkinson, D. Y., MacDowell, A. A. \& Balsara, N. P. Detection of subsurface structures underneath dendrites formed on cycled lithium metal electrodes. Nat. Mater. 13, 69-73 (2014).

9 Niitani, T., Shimada, M., Kawamura, K., Dokko, K.,. Rho, Y.-H. \& Kanamura, K. Synthesis of $\mathrm{Li}^{+}$ion conductive PEO-PSt block copolymer electrolyte with microphase separation structure. Electrochem. Solid State Lett. 8, A385-A388 (2005).

10 Singh, M., Odusanya, O., Wilmes, G. M., Eitouni, H. B., Gomez, E. D., Patel, A. J., Chen, V. L., Park, M. J., Fragouli, P., latrou, H., Hadjichristidis, N., Cookson, D. \& Balsara, N. P. Effect of molecular weight on the mechanical and electrical properties of block copolymer electrolytes. Macromolecules 40, 4578-4585 (2007).

11 Panday, A., Mullin, S., Gomez, E. D., Wanakule, N., Chen, V. L., Hexemer, A., Pople, J. \& Balsara, N. P. Effect of molecular weight and salt concentration on conductivity of block copolymer electrolytes. Macromolecules 42, 4632-463 (2009).

12 Choi, I., Ahn, H. \& Park, M. J. Enhanced performance in lithium-polymer batteries using surface-functionalized $\mathrm{Si}$ nanoparticle anodes and self-assembled block copolymer electrolytes. Macromolecules 44, 7327-7334 (2011).

13 Young, W. S. \& Epps, T. H. Ionic conductivities of block copolymer electrolytes with various conducting pathways: sample preparation and processing considerations. Macromolecules 45, 4689-4697 (2012).

14 Yuan, R., Teran, A. A., Gurevitch, I., Mullin, S. A., Wanakule, N. S. \& Balsara, N. P. Ionic conductivity of low molecular weight block copolymer electrolytes. Macromolecules 46, 914-921 (2013).

15 Bouchet, R., Phan, T. N. T., Beaudoin, E., Devaux, D., Davidson, P., Bertin, D. \& Denoyel, R. Charge transport in nanostructured PS-PEO-PS triblock copolymer electrolytes. Macromolecules 47, 2659-2665 (2014).

16 Chintapalli, M., Chen, X. C., Thelen, J. L., Teran, A. A., Wang, X., Garetz, B. A. \& Balsara, N. P. Effect of grain size on the ionic conductivity of a block copolymer electrolyte. Macromolecules 47, 5424-5431 (2014).

17 Rolland, J., Brassinne, J., Bourgeois, J. P., Poggi, E., Vlad, A. \& Gohy, J. F. Chemically anchored liquid-PEO based block copolymer electrolytes for solid-state lithium-ion batteries. J. Mater. Chem. A 2, 11839-11846 (2014).

18 Zardalidis, G., Gatsouli, K., Pispas, S., Mezger, M. \& Floudas, G. Ionic conductivity, self-assembly, and viscoelasticity in poly(styrene-b-ethylene oxide) electrolytes doped with LiTf. Macromolecules 48, 7164-7171 (2015).

19 Cho, B. K., Jain, A., Gruner, S. M. \& Wiesner, U. Mesophase structure-mechanical and ionic transport correlations in extended amphiphilic dendrons. Science 305, 1598-1601 (2004).
20 Khurana, R., Schaefer, J. L., Archer, L. A. \& Coates, G. W. Suppression of lithium dendrite growth using cross-linked polyethylene/polyethylene oxide electrolytes: A new approach for practical lithium-metal polymer batteries. J. Am. Chem. Soc. 136, 7395-7402 (2014)

21 Nagaoka, K., Naruse, H., Shinohara, I. \& Watanabe, M. High ionic conductivity in poly (dimethyl siloxane-co-ethylene oxide) dissolving lithium perchlorate. J. Polym. Sci., Polym. Lett. Ed.22, 659-663 (1984).

22 Fonseca, C. P. \& Neves, S. Characterization of polymer electrolytes based on poly (dimethyl siloxane-co-ethylene oxide). J. Power Sources 104, 85-89 (2002).

23 Ryu, S.-W. Effect of lithium ion concentration on thermal properties in novel single-ion polymer electrolyte. Polymer J. 40, 688-693 (2008).

24 Jo, G., Jeon, H. \& Park, M. J. Synthesis of polymer electrolytes based on Poly(ethylene oxide) and an anion-stabilizing hard polymer for enhancing conductivity and cation transport. ACS Macro Lett 4, 225-230 (2015).

25 Doyle, M., Fuller, T. F. \& Newman, J. Modeling of galvanostatic charge and discharge of the lithium/polymer/insertion cell. J. Electrochem. Soc. 140, 1526-1533 (1993).

26 Wang, C., Appleby, A. J. \& Little, F. E. Electrochemical impedance study of initial lithium ion intercalation into graphite powders. Electrochim. Acta 46, 1793-1813 (2001).

27 Zhang, S., Ding, M. S., O, K., Xu, K., Allen, J. \& Jow, T. R. Understanding solid electrolyte interface film formation on graphite electrodes. Electrochem. Solid-State Lett. 4, A206-A208 (2001).

28 Barbier, D., Brown, D., Grillet, A.-C. \& Neyertz, S. Interface between end-functionalized PEO oligomers and a silica nanoparticle studied by molecular dynamics simulations. Macromolecules 37, 4695-4710 (2004).

29 Zhao, J., Lu, Z., Wang, H., Liu, W., Lee, H. W., Yan, K., Zhuo, D., Lin, D., Liu, N. \& Cui, Y. Artificial solid electrolyte interphase-protected LixSi nanoparticles: an efficient and stable prelithiation reagent for lithium-ion batteries. J. Am. Chem. Soc. 137, 8372-8375 (2015).

30 Jo, G., Ahn, H. \& Park, M. J. Simple route for tuning the morphology and conductivity of polymer electrolytes: one end functional group is enough. ACS Macro Lett. 2, 990-995 (2013).

31 Kim, C. K., Won, J., Kim, H. S., Kang, Y. S., Li, H. G. \& Kim, C. K. Density functional theory studies on the dissociation energies of metallic salts: relationship between lattice and dissociation energies. J. Comput. Chem. 22, 827-834 (2001).

32 Young, W.-S. \& Epps, T. H. III Salt doping in PEO-containing block copolymers: counterion and concentration effects. Macromolecules 42, 2672-2678 (2009).

33 Panday, A., Mullin, S., Gomez, E. D., Wanakule, N., Chen, V. L., Hexemer, A., Pople, J. \& Balsara, N. P. Effect of molecular weight and salt concentration on conductivity of block copolymer electrolytes. Macromolecules 42, 4632-4637 (2009).

$34 \mathrm{Lu}$, X. \& Weiss, R. A. Phase behavior of blends of poly(ethylene glycol) and partially neutralized poly(acrylic acid). Macromolecules 28, 3022-3029 (1995).

35 Lutkenhaus, J. L., Hrabak, K. D., McEnnis, K. \& Hammond, P. T. Elastomeric flexible free-standing hydrogen-bonded nanoscale assemblies. J. Am. Chem. Soc. 127, 17228-17234 (2005).

36 Sax, J. \& Ottino, J. M. Modeling of transport of small molecules in polymer blends application of effective medium theory. Polym. Eng. Sci. 23, 165-176 (1983). 